\title{
Breast cancer and postmenopausal obesity: the risk factors in this relationship
}

\author{
Clarissa Germano Barp ${ }^{1}$, Durinézio Jose de Almeida ${ }^{2}$, Guilherme Barroso Langoni de Freitas ${ }^{1,3}$ \\ ${ }^{1}$ Department of Pharmacy, State University of Center-West, Camargo Varela de Sá, 03, Vila Carli, Guarapuava, Paraná, Brazil \\ ${ }^{2}$ Department of Biology, State University of Maringá. Colombo Avenue, 5790, Zone 07, Maringá, Paraná, Brazil \\ ${ }^{3}$ Department of Medicine, Federal University of Paraná (UFPR), Internal Medicine Program, General Carneiro, 181, Alto da Glória, \\ Curitiba, Paraná, Brazil
}

\section{Email address:}

Prof.gbarroso@yahoo.com.br (Barp C. G.)

\section{To cite this article:}

Clarissa Germano Barp, Durinézio Jose de Almeida, Guilherme Barroso Langoni de Freitas. Breast Cancer and Postmenopausal Obesity: the Risk Factors in this Relationship. Cancer Research Journal. Vol. 2, No. 1, 2014, pp. 9-14. doi: 10.11648/j.crj.20140201.12

\begin{abstract}
The prevalence of obesity is increasing rapidly in both the developed and the developing countries, thus contributing to the occurrence of other diseases, e.g. hypertension, diabetes and breast cancer. Breast cancer involves a multifactorial process in which the surrounding microenvironment is known to be of great importance in the development and progression of the disease. Thus, adipose tissue, due to its immunological, metabolic and endocrine properties, may play a crucial role in the onset and progression of the disease. The relationship between obesity and breast cancer needs to be studied and characterized so that more effective treatments can be developed, faster and more secure diagnostic and prognostic tests might emerge and, mainly, so that public policies can be designed to prevent the increasing incidence of the disease related to obesity. The main hypotheses regarding the increased risk of developing breast cancer among obese women, i.e. sex hormones, adipokines, insulin and cytokines, are discussed in this review.
\end{abstract}

Keywords: Obesity, Breast cancer, Adipokines, Sex hormones, Cytokines, Insulin

\section{Introduction}

Obesity is excess weight caused by a buildup of fatty tissue that can lead to poor health. It is increasingly prevalent and has multifactorial triggers, including genetic, and environmental factors, and mainly humoral and neural mechanisms that control appetite and satiety $[1,2]$. The condition is associated with an increased incidence of various diseases such as cardiovascular diseases, diabetes mellitus and cancer, especially esophageal, pancreatic, colorectal, kidney, endometrial and breast cancer [3].

Breast cancer is the neoplastic disease that most affects women and is among the cancers with the highest rates of incidence in the world, with rates of 1,677 million new cases and 522,000 deaths per year [4]. It involves a multifactorial process [5] and has several predisposing factors, among which are early menarche, genetic factors, familial predisposition, obesity and post-menopause hormone replacement $[6,7]$.

Studies show that the body mass index (BMI) may indicate a worse prognosis in postmenopausal women, since overweight postmenopausal women were found to have a
$25 \%$ increase in the risk developing breast cancer. In addition to the higher risk of developing the disease, studies suggest that obese women with breast cancer have $250 \%$ more chance of treatment failure when compared to lean women $[8,9]$.

Therefore, it is essential to characterize which metabolic pathways and mediators are involved in the obesity-breast cancer relationship. Hence, this review has sought to characterize how the physiological changes caused by excess fatty tissue in postmenopausal women are related to the onset and/or development of mammary tumors.

\section{Hypotheses Regarding the Obesity-Breast Cancer Relationship}

There are several indications that the microenvironment surrounding the tumor is not passive, and is recognized as being active in tumor progression. In breast cancer, that microenvironment is comprised of endothelial and immune cells, fibroblasts and adipocytes [10].

Obesity and its physiological changes may be the path to explaining its relationship to breast cancer. This is because 
adipose tissue is not only involved in thermal protection and energy deposition; it also plays a physiological role in cells, with important functions in the immune, metabolic and endocrine systems which are described below [3].

\subsection{The Estrogen Hypothesis}

The development of breast cancer is known to involve the action of hormonal factors, particularly estrogen. Thus, one hypothesis for the emergence of the disease is the excess production of estrogens, which leads to a transition from hyperplasia to neoplasia, or stimulation of cell proliferation [11]. In women of reproductive age, the formation of biologically active estrogen, estradiol (E2), occurs directly in peripheral tissues through the conversion of circulating androstenedione (A), produced by the adrenal glands or ovaries, or by the conversion of androstenedione (A) to estrone (E1) in target tissues, where it is finally converted to estradiol (E2) (Figure 1).

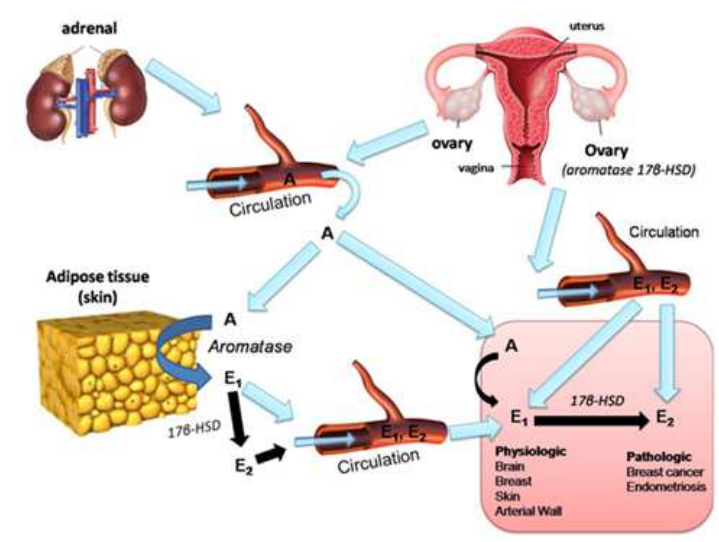

Figure 1. Biosynthesis of estrogen hormones and organ targets.

Androgenic steroids are metabolized to estrogens by the action of an aromatase enzyme complex that, in premenopausal women, is expressed, mainly, in the ovaries. However, following menopause, a larger proportion of estrogen is produced in the adipose tissue, due to aromatization of the androstenedione precursor (produced by the adrenal glands and the ovaries) to estrone (E1), finally forming estradiol (E2), by the action of the estradiol-17 $\beta$ hydroxysteroid dehydrogenase (17 $\beta$ - HSD) enzyme [12]. Aromatase activity is increased in obesity due to the induction of the enzyme promoters and results in the increased formation of estradiol.

There are two known estrogen receptors, $\alpha$ and $\beta$ (ER $\alpha$ and ER $\beta$ ), which are nuclear-type receptors found in several cellular compartments, including the cell membrane, cytosol, nucleus and mitochondria. They are capable of modulating the transcription of genes that present hormone-specific responses. These receptors are encoded by different genes, ESR1 and ESR2, respectively, and exhibit high levels of homology in the DNA binding site. However, they differ significantly in the N-terminal portion, which is a factor related to the production of several different physiological responses. Furthermore, ER $\alpha$ and ER $\beta$ have different distribution patterns in the tissues, suggesting that an imbalance in these receptors might be involved in the development of diseases such as cancer $[13,14]$.

Estradiol-17 $\beta$ (E2) binds to both receptors with high affinity, however, some metabolites show greater selectivity for one or the other receptor. Moreover, some ER $\beta$ promoters can act as negative modulators of ER $\alpha$. This difference between the estrogen receptors has allowed the study of selective molecules for the treatment of breast cancer. This is because studies have shown that ER $\alpha$ has a proliferative action, while ER $\beta$ is seen as a possible suppressor of the disease $[15,16]$.

The $\beta$ receptor $(\mathrm{ER} \beta)$ is known to influence breast cancer in various ways, because it suffers the action of different ligands, such as estrogens and antiestrogens, which activate genomic and non-genomic pathways. In non-genomic activation, the receptor interacts with membrane proteins, leading to the kinase activation. In genomic activation, receptor dimerization occurs with the subsequent translocation of the receptor from the cytoplasm to the nucleus or mitochondria, promoting its binding to estrogen response factors and, thus, to its acting in regulatory regions. Thus, some isoforms of ER $\beta$, such as ER $\beta 2$ and nuclear ER $\beta 5$ are associated with a good prognosis, while others, such as cytoplasmic ER $\beta 2$ are related to a decreased chance of survival [16].

Obese patients also have lower concentrations of sex hormone-binding globulin (the protein responsible for transporting estrogen), hence the amount of free estradiol in the bloodstream is increased, consequently increasing its biological activity. This endocrine change contributes to the increased risk of developing breast cancer in obese postmenopausal women [17].

\subsection{The Adipokine Hypothesis}

Adipose tissue also has the function of secreting other compounds, the adipokines, which include leptin, adiponectin, interleukins and resistin. Leptin is a hormone involved in appetite regulation, immunity, differentiation and the proliferation of different cell types. In the breast, it is necessary for normal development and for lactation. In breast cancer, both the leptin and its receptors (ObR) are overexpressed. Additionally, the functional binding of leptin to signaling molecules occurs, which exacerbates its function and the activation of certain signaling pathways $[18,19,20]$. Leptin acts by stimulating DNA synthesis and cell growth by activating signaling cascades involved in tumor development, including the janus kinase pathway (JAK) (STAT 3 activation) and the Akt/GSK3 pathway. Giordano et al (2012) highlight the fact that leptin increases the expression of the Hsp90 protein (heat shock protein 90), which is responsible for the increase in the level of Her2 (human epidermal growth factor receptor 2). Additionally, leptin is also involved in the increased activation of estrogen and negatively regulates apoptosis [21,9].

Adiponectin increases the catabolism of fatty acids, insulin sensitivity, inhibits TNF $\alpha$ (increasing the expression 
of A20 gene, a suppressor of TNF receptor-associated factor (TRAF) 1), and also displays anti-inflammatory features. It has been characterized as an antiproliferative agent in tumor cell growth, because it represses genes involved in cell activation and the metabolism of fatty acids. Thus, it has been identified as a factor inversely proportional to the development of cancer [22]. However, in most cases, adiponectin levels are decreased in obesity $[9,23]$. This imbalance in the secretion of adipokines by fat cells may explain the relationship with breast cancer.

\subsection{The Cytokine Hypothesis}

As mentioned above, adipocytes are cells that play an endocrine role, producing hormones, cytokines and growth factors. Cytokines are released from adipocyte precursor cells, called preadipocytes, to provide a suitable microenvironment for cytokine proliferation. In obesity, the maturation of these cells is unbalanced, with larger amounts of pre-adipocytes, and a resulting increase in the secretion of cytokines, which together with the increased secretion of leptin stimulates the formation of macrophages, leading to the production of proinflammatory cytokines and pro-angiogenic factors [24].

Interleukin-6 (IL-6) is produced by fibroblasts, macrophages and lymphocytes and has been detected in significant concentrations in samples of breast tumor. In chronic inflammation, which is present in obesity, IL-6 has various physiological effects. Of note among them are its proinflammatory effects, the regulation of genes involved in cellular proliferation, survival and differentiation, the promotion of tumor growth due to angiogenic and anti-apoptotic actions and also the increased production of $17 \beta-H S D$ [25]. However, these known systemic actions of IL-6 are in contrast to the results of recent studies into its local action, which are related to a positive prognosis. This is because its autocrine and paracrine action has been shown to play a role in controlling cell growth in ER positive and precursor cell cancer cells, as well as controlling metastasis. Thus, in the future, the local application of IL-6 may be a tool for the treatment of breast cancer [26]. Therefore, it is important to conduct new studies on the actual effects of IL-6 so that new treatments can be rationally targeted.

TNF is another important cytokine. It is involved in inflammation, tissue repair and immune reactions, and induces both necrosis and apoptosis in tumor cells [27]. It is produced by macrophages, $\mathrm{T}$ lymphocytes and natural killer cells and acts by binding to two receptors, TNFR1 and TNFR2. TNFR1 is responsible for most of the biological activity and has a death domain (DD) that is absent in TNFR2. This domain provides the ability to induce cell death by apoptosis, while it also translates cell survival signals. Following TNF $\alpha$ binding, the receptor trimerizes and releases the DD protein, triggering a chain of signals that activate proteases responsible for apoptosis, which activate endonucleases that will fragment the DNA. However, when there is activation of survival signals by the TNFR1 receptor, TNFR-associated factor 2 (TRAF2) is recruited into the complex formed due to trimerization of the receptor, thus inhibiting apoptosis via the cellular inhibitor of apoptosis protein (cIAP). The most important signaled events are activation of the nuclear factor kappa B (NF-KB), transcription of the factor via the NF-kB kinase (NIK) inductor and of the $\mathrm{kB}$ kinase complex (IKK) inhibitor which induces the transcription of anti-apoptotic, proliferative, immunomodulatory and inflammatory genes. NF-kB is an important transcription factor in the prevention of TNF-mediated apoptosis. Therefore, its inhibition may increase the effectiveness of treatments that induce apoptosis [28]. Particularly in breast cancer, it has been observed that TNF $\alpha$ induces apoptosis and reduces proliferation in some cell lines, while others have been shown to be resistant to apoptosis induced by the cytokine [29].

Prostaglandin E2 (PGE2) is the product derived from the enzyme cyclooxygenase-2 (COX -2) that is found in the largest amounts in tumors. It acts as an inflammatory factor, being secreted in an autocrine/paracrine manner, and acts on the ER1-4 receptors through receptors coupled to the G-protein. A relationship has been shown to exist between activation of the COX-2/PGE2 pathway and increased activity of the estrogen-producing enzyme (17ß-HSD), which can lead to an increase in the development of breast cancer. Activation of this pathway is involved in events such as increased angiogenesis, invasion and metastasis [30,31]. The E2 and E4 receptors are seen as being those most involved in the development of breast cancer, because they are related to the expression of the aromatase enzyme and of cancer cells, acting through the cAMP/PKA pathway, which is also involved in the increase in VEGF [32]. Calcitriol (vitamin D) has been referred to as a preventative and therapeutic factor in breast cancer, as it negatively regulates COX-2, with a consequent decline in the concentration of PGE2 and decreased activation of the MAPK pathway [30]. Hence, vitamin $\mathrm{D}$ is being studied as a tumor suppressor and may represent a possible treatment option and means of preventing the disease [33].

Thus, the increase in the release of proinflammatory cytokines, resulting from obesity is a strong risk factor for the development of breast cancer in postmenopausal women.

\subsection{The Testosterone/Androgen Hypothesis}

In post-menopausal Western women, the increase in the levels of androgens, particularly testosterone, is determined by an excess of adipose tissue, insulin resistance and increased circulating insulin [34]. The influence of androgenic hormones on the development of breast cancer is still unclear. Most studies show that high levels of androgens are positively involved in the development of the cancer, while others support the idea that androgens inhibit the development of the disease.

A positive association is justified by the presence of large numbers of androgen receptors (AR) in breast tissue in which cancer present, where androgens act directly on their 
receptor, resulting in growth and proliferation [35]. Another circumstance concerns the testosterone precursor molecules androstenedione and dehydroepiandrosterone (DHEAS), which also serve as precursors for the formation of estradiol through the action of the aromatase enzyme, which, in the post-menopause mainly occurs in adipose tissue, with aromatization being the indirect contribution to the development of the cancer. With the increase in adipose tissue there is also an increase in the induction of aromatase promoters, resulting in increased estrogen production [36,37]. Furthermore, some authors have shown that DHT increases cell proliferation in the MCF-7 and MDA-MB-453 cell lines [38]. These cellular models are characterized as breast cancer cell lines because they can express androgen receptors and measure cell growth based on androgenic activity. MCF-7 is estrogen receptor-alpha-positive (ER-alpha-positive), weakly invasive and luminal epithelial-like, while MDA-MB231 is ER-alpha-negative, highly invasive and fibroblast-like. Essentially, they express low and high PKC activities, respectively.

By contrast, the possibility of androgens acting to counteract the development of breast cancer is suggested by the indication that testosterone and dihydrotestosterone (DHT) negatively influence mitogenic activity [37,39]. The antiproliferative effects were seen in the increase in the proportion of cells in the G0/G1 phase in the MCF-7 cell line, in addition to results showing a decrease in ZR75-1 tumor cells in athymic mice following treatment with DHT. DHT also seems to demonstrate anti-apoptotic activity by negatively regulating the protooncogene $\mathrm{Bcl}-2$ pathway, by acting on AR receptors [37,38].

\subsection{The Insulin and Insulin-Like Growth Factor (IGF)-I Hypothesis}

Insulin and IGF-1 and -2 (insulin-like growth factor 1 and 2) are hormones and peptide growth factor homologs that perform functions in the control of cellular development, including cellular proliferation, differentiation and apoptosis, and also play a physiological role, acting on growth, metabolism and longevity. Obesity is known to lead to insulin resistance, and this condition causes the pancreas to compensate for the excess free glucose by increasing insulin production, with a consequent increase in plasma insulin levels [40].

The relationship between breast cancer and insulin may be explained by this compensatory mechanism, due to the hormone acting as a tissue growth factor, being related to the increased proliferation of tumor cells. Insulin may act by directly signaling through its receptors (such as the insulin-A receptor) or indirectly through growth factors such as IGFs and estradiol. The excess free glucose also influences the propensity to develop breast cancer, due to the fact that tumor cells use it to proliferate $[40,41,42]$. Furthermore, the excess of insulin leads to decreased production of SHBG in the liver, increasing the bioavailability of testosterone and estradiol [34].

The IGF-1R subtype receptors seem to play a crucial role in the transformation and maintenance of tumor cells by enhancing cell survival, with protection from apoptosis via multiple pathways. Thus, the insulin-like growth factors (IGFs) are able to modulate cell adhesion and migration processes [40]. In addition, the insulin-A receptor has a strong mitogenic activity [34].

Thus, drugs used to treat type 2 diabetes, such as metformin, are of great interest as possible treatment options for breast cancer. Metformin may act both directly and indirectly as an anti-cancer agent. Directly on the cancer cells by inhibiting the respiratory chain in the mitochondria and indirectly by acting on AMP-activated protein kinase, suppressing gluconeogenesis, with a consequent reduction in circulating insulin [43]. The insulin receptor (IR) is a kinase receptor that is activated by insulin with subsequent stimulation of intracellular biosynthesis of testosterone.

\section{Conclusion}

The current increase in global levels of obesity has been shown to influence the formation and progression of breast cancer in post-menopausal women. The microenvironment surrounding the tumor has a great influence on the course of the disease. Therefore it is essential to understand the mechanisms involved so that strategies for prevention and treatment of disease can be developed. Many studies have suggested adipose tissue is a predisposing factor in the formation and development of the tumor through metabolic, hormonal and immunological pathways. This review highlighted the main hypotheses, i.e. the insulin and insulin-Like growth factor, testosterone/androgen, cytokine, adipokine and estrogen hypotheses. Advances in genetic research should elucidate some uncertainties about the role of hormones and the predisposition to obesity and risk factors.

\section{References}

[1] Robbins \& Cotran - Patologia - Bases Patológicas das

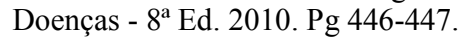

[2] Undurti N D; M.D.; F.A.M.S. (2010) Obesity: Genes, brain, gut, and environment. Nutrition 26, 459-473.

[3] Doyle S L, Donohoe C L, Lysaght J, Reynolds J V (2012) Symposium 3: Obesity-related cancers - Visceral obesity, metabolic syndrome, insulin resistance and câncer. P Nutr Soc. 71, 181-189.

[4] http://www.cancerresearchuk.org/cancer-info/cancerstats/rep orts/

[5] C. Pichard C, Plu-Bureau G, Neves e Castro M, Gompel A. Insulin resistance, obesity and breast cancer risk. Maturitas. Volume 60, Issue 1, 20 May 2008, Pages 19-30.

[6] Hancke K, Grubeck D, Hauser N, Kreienberg R, Weiss JM. Adipocyte fatty acid-binding protein as a novel prognostic factor in obese breast cancer patients. Breast Cancer Res Treat (2010) 119:367-377. 
[7] Lautenbach A, Budde A, Wrann C D, Teichmann B, Vieten G, Karl T, Nave H (2009) Obesity and the Associated Mediators Leptin, Estrogen and IGF-I Enhance the Cell Proliferation and Early Tumorigenesis of Breast Cancer Cells. Nutr Cancer 61(4), 484-491.

[8] Bergstrom A, Pisani P, Tenet V, Wolk A, Adami H (2001) Overweight as an avoidable cause of cancer in europe. Int $\mathrm{J}$ Cancer: 91, 421-430.

[9] Jardé T, Perrier S, Vasson M, Caldefie-Chézet F (2011) Molecular mechanisms of leptin and adiponectin in breast câncer. Eur J Cancer 47, 33-4 3.

[10] Place A E, Huh S J, Polyak K (2011) The microenvironment in breast câncer progression: biology and implications for treatment. Breast Cancer Res, 13:227.

[11] Clamp A, Danson S, Clemons M (2003) Hormonal and genetic factors for breast cancer risk. J Roy Coll Surg Edin 23-31.

[12] Bellemare V, Laberge P, Noël S, Tchernof A, Luu-The V (2009) Differential estrogenic 17_-hydroxysteroid dehydrogenase activity and type 12 17-hydroxysteroid dehydrogenase expression levels in preadipocytes and differentiated adipocytes. J Steroid Biochem 114, 129-134.

[13] Kumar S, Lata K, Mukhopadhyay S, Mukherjee T K (2010) Role of estrogen receptors in pro-oxidative and anti-oxidative actions of estrogens: A perspective. Biochim Biophys Acta $1800,1127-1135$.

[14] Carruba G (2007) Estrogen and Prostate Cancer: An Eclipsed Truth in na Androgen-Dominated Scenario. J Cell Biochem 102, 899-911.

[15] Helguero L A, Faulds M H, Gustafsson J K, Haldosén L (2005) Estrogen receptors alfa (ERa) and beta (ERb) differentially regulate proliferation and apoptosis of the normal murine mammary epithelial cell line HC11. Oncogene 24, 6605-6616.

[16] Leung Y, Lee M, Lam H, Tarapore P, Ho S (2012) Estrogen receptor-beta and breast cancer: Translating biology into clinical practice. Steroids 77, 727-737.

[17] Rose D.P, Vona-Davis L (2010) Interaction between menopausal status and obesity in affecting breast cancer risk. Maturitas 66, 33-38.

[18] Jarde T, Caldefie-Chezet F, Damez M, Mishellany F, Penault-Llorca F, Guillot J, Vasson M P (2008) Leptin and leptin receptor involvement in cancer development: A study on human primary breast carcinoma. Oncol Rep 19, 905-911.

[19] Giordano C, Vizza D, Panza S, Barone I, Bonofiglio D, Lanzino M, Sisci D, Amicis F De, Fuqua S A W, Catalano S, Ando S (2012) Leptin increases HER2 protein levels through a STAT3-mediated up-regulation of Hsp90 in breast cancer cells. Mol Oncol 1-13.

[20] Marotta L L C, Almendro V, Marusyk A, Shipitsin M, Schemme J, Walker S R, Bloushtain-Qimron N, Kim J J, Choudhury S A, Maruyama R, Wu Z, Gönen M, Mulvey L A, Bessarabova M O, Huh S J, Silve S J, Kim S Y, Park S Y, Lee H E, Anderson K S, Richardson A L, Nikolskaya T, Nikolsky Y, Liu X S, Root D E, Hahn W C, Frank D A, Polyak K (2011) The JAK2/STAT3 signaling pathway is required for growth of CD44+CD24- stem cell- like breast cancer cells in human tumors. J Clin Invest http://www.jci.org Volume 121 Number 7.
[21] Garofalo C, Koda M, Cascio S (2006) Increased Expression of Leptin and the Leptin Receptor as a Marker of Breast Cancer Progression: Possible Role of Obesity-Related Stimuli. Clin Cancer Res 12, 1447-1453.

[22] Ramos-Nino ME. The Role of Chronic Inflammation in Obesity-Associated Cancers. ISRN Oncology. Volume 2013 (2013), Article ID 697521, 25 pages.

[23] Housa D, Housová J, Vernerová Z, Haluzík M (2006) Adipocytokines and Câncer. Physiol Res 55, 233-244.

[24] Gilbert C A, Slingerland J M (2013) Cytokines, Obesity, and Cancer: New Insights on Mechanisms Linking Obesity to Cancer Risk and Progression. Annu Rev Med 64, 45-57.

[25] Nicolini A, Carpi A, Rossi G (2006) Cytokines in breast câncer. Cytokine Growth F R 17, 325-337.

[26] Dethlefsen C, Højfeldt G, Hojman P (2013) The role of intratumoral and systemic IL-6 in breast câncer. Breast Cancer Res Tr 138, 657-664.

[27] Bogin L, Papa M Z, Polak-Charcon S, Degani H (1998) TNF-induced modulations of phospholipid metabolism in human breast cancer cells. Biochim Biophys Acta 1392, 217-232.

[28] Horssen R V, Hagen T L M T (2006) Eggermont, A. M. M. TNF- $\alpha$ in Cancer Treatment: Molecular Insights, Antitumor Effects, and Clinical Utility. The Oncologist 11, 397-408.

[29] Rivas M A, Carnevale R P, Proiettia C J, Rosemblit C, Beguelin W, Salatino M, Charreau E H, Frahm I, Sapia S, Brouckaert P, Elizalde P V, Schillaci R (2008) TNF $\alpha$ acting on TNFR1 promotes breast cancer growth via $\mathrm{p} 42 / \mathrm{P} 44$ MAPK, JNK, Akt and NF- $\kappa B$-dependent pathways. Exp Cell Res 314, 509-529.

[30] Yuan L, Jiang R, Yang Y, Ding S, Deng H (2012) 1,25-Dihydroxyvitamin D3 inhibits growth of the breast cancercell line MCF-7 and downregulates cytochrome P4501B1through the COX-2/PGE2 pathway. Oncol Rep 28, 2131-2137.

[31] Holt D, Ma X, Kundu N, Collin P D, Fulton A M (2012) Modulation of host natural killer cell functions in breast câncer via prostaglandin E2 receptors EP2 and EP4. J Immunother 35(2), 179-188.

[32] Reader J, Holt D, Fulton A (2011) Prostaglandin E2 EP Receptors as Therapeutic Targets in Breast Câncer. Cancer Metast Rev 30(0), 449-463.

[33] Thill M, Hoellen F, Becker S, Dittmer C, Fischer D, Kummel S, Salehin D, Friedrich M, Koster F, Diedrich K, Cordes T (2012) Expression of Prostaglandin- and Vitamin D-metabolising Enzymes in Benign and Malignant Breast Cells. Anticancer Res 32, 367-372.

[34] Campagnoli C, Pasanisi C, Castellano I, Abba` C, Brucato T, Berrino F (2013) Postmenopausal breast cancer, androgens, and aromatase inhibitors. Breast Cancer Res Tr 139, 1-11.

[35] Dimitrakakis C, Bondy C. Androgens and the breast. Breast Cancer Res 2009, 11:212.

[36] Aspinall S R, Cook D B, Shenton B K, Griffiths A.B, Griffith C D M, Bliss R D, Lennard T W J (2003) Serum adrenal androgens in women with primary operable breast cancer and their relationship with age and body mass index. Breast 12, 63-71. 
[37] Somboonporn W, Davis S R (2004) Postmenopausal testosterone therapy and breast cancer risk. Maturitas 49, 267-275.

[38] Suzuki T, Miki Y, Takagi K, Hirakawa H, Moriya T, Ohuchi N, Sasano H (2010) Androgens in human breast carcinoma. Med Mol Morphol 43, 75-81.

[39] Tiefenbacher K, Daxenbichler G. The Role of Androgens in Normal and Malignant Breast Tissue. Breast Care (Basel). 2008;3(5):325-331. Epub 2008 Oct 16.

[40] Perks C M, Holly J M P (2011) Mechanisms Underlying the Relationship Between Obesity and Breast Câncer. Endocrin Metab Clin 40, 485-507.
[41] Patterson R E, Rock C L, Kerr J, Natarajan L, Marshall S J, Pakiz B, Cadmus-Bertram L A (2013) Metabolism and Breast Cancer Risk: Frontiers in Research and Practice. J Acad Nutr Diet 113, 288-296.

[42] Vecchia C, Giordano S H, Hortobagyi G N, Chabner B (2011) Overweight, Obesity, Diabetes, and Risk of Breast Cancer: Interlocking Pieces of the Puzzle. The Oncologist 16, 726-729.

[43] Goodwin P J, Stambolicb V (2011) Obesity and insulin resistance in breast cancer-Chemoprevention strategies with a focus on metformin. The Breast 20, S3, S31-S35. 